\title{
Pour-on Container
}

National Cancer Institute

\section{Source}

National Cancer Institute. Pour-on Container. NCI Thesaurus. Code C149766.

Container with built-in applicator for pouring-on use. 